BULL. AUSTRAL. MATH. SOC.

VOL. 31 (1985), 445-450.

\title{
THE FARKAS LEMMA OF \\ SHIMIZU, AIYOSHI AND KATAYAMA
}

\section{Charles Swartz}

\begin{abstract}
Shimizu, Aiyoshi and Katayama have recently given a finite dimensional generalization of the classical Farkas Lemma. In this note we show that a result of Pshenichnyi on convex programming can be used to give a generalization of the result of Shimizu, Aiyoshi and Katayama to infinite dimensional spaces. A generalized Farkas Lemma of Glover is also obtained.
\end{abstract}

In [7], Theorem 2.1, Shimizu, Aiyoshi and Katayama have given a finite dimensional generalization of the classical lemma of Farkas ([2]). In this note we show that an infinite dimensional generalization of the result of Shimizu, Aiyoshi and Katayama can be obtained from a global characterization of a minimum in a convex programming problem due to Pshenichnyi ([6], II.2.1). We also show that a generalized Farkas Lemma due to Glover can be obtained from Pschenichnyi's result ([3], Theorem 1).

We begin by describing the result of Shimizu, Aiyoshi and Katayama. Throughout this paper, let $X$ be a (real) Hausdorff locally convex topological vector space with dual $X^{\prime}$ and duality form $($,$) . We$ assume throughout that $X^{\prime}$ is equipped with the weak* topology from $X$. Let $A$ and $B$ be compact subsets of $X$ such that 0 is not in the convex hull of $A(0 \$ \operatorname{co} A)$. The result of Shimizu, Aiyoshi and

Received 30 November 1984.

Copyright Clearance Centre, Inc. Serial-fee code: 0004-9727/85 $\$ \mathrm{~A} 2.00+0.00$. 
Katayama ([7], 2.1) then states that in a finite dimensional space the following conditions are equivalent:

(1) $\max \left\{\left\langle x^{\prime}, b\right\rangle: b \in B\right\} \leq 0$ implies $\max \left\{\left\langle x^{\prime}, a\right\rangle: a \in A\right\} \geq 0$;

(2) there exist $x_{0} \in \operatorname{co} A, x_{1} \in \operatorname{co} B$ and $t \leq 0$ such that $x_{0}=t x_{1}$

If $E$ is a subset of $X$, let $S(\cdot \mid E)$ be the support functional of $E, S\left(x^{\prime} \mid E\right)=\sup \left\{\left\langle x^{\prime}, x\right\rangle: x \in E\right\}$ for $x^{\prime} \in X^{\prime} \quad([1], 2.1 .3)$. Note that $S(\cdot \mid E)=S(\cdot \mid \overline{c o} E)$, where $\overline{\text { co }} E$ is the closed convex hull of $E$. Using the notation of support functional, (1) becomes:

$$
S\left(x^{\prime} \mid B\right) \leq 0 \text { implies } S\left(x^{\prime} \mid A\right) \geq 0 \text {. }
$$

Now the support functional is always convex so this suggests replacing condition ( 1 ) by the condition:

(I') $h(x) \leq 0$ implies $f(x) \geq 0$,

where $h$ and $f$ are convex functions defined on $X$. We then need to obtain an appropriate generalization of (2) for this situation. This is given in Theorem 2 (also Corollary 3 ).

We now fix the notation and terminology which will be used. Let $h$ and $f$ be real valued convex functions defined on $X$. The subgradient of $f$ at $x_{0}$ is $\partial f\left(x_{0}\right)=\left\{x^{\prime} \in X^{\prime}: f(x)-f\left(x_{0}\right) \geq\left\langle x^{\prime}, x-x_{0}\right\rangle\right.$ for $\left.x \in X\right\}$ ([6], I.1.1; [4], 6D). If $f$ is continuous at $x_{0}$, then $\partial f\left(x_{0}\right)$ is a non-void, weak ${ }^{*}$ compact subset of $X^{\prime}$ ([4], I4B). A similar statement holds if $f$ is a lower semicontinuous sublinear function $(f$ is sublinear if $f(x+y) \leq f(x)+f(y)$ and $f(t x)=t f(x)$ for $x, y \in X$ and $t \geq 0)$; that is, $\partial f\left(x_{0}\right)$ is a non-void weak * compact subset of $X^{\prime}$ ([10], Proposition 1 and [1], .4).

If $\Omega$ is a subset of $X$ and $x_{0} \in \Omega$, then the cone of feasible directions to $\Omega$ at $x_{0}$ is

$$
F\left(\Omega, x_{0}\right)=\left\{e: \text { there exists } \alpha>0 \text { such that } x_{0}+t e \in \Omega \text { for } 0 \leq t \leq \alpha\right\}
$$

([6], II; [4], 14E). For a convex set, 


$$
F\left(\Omega, x_{0}\right)=\left\{t\left(x-x_{0}\right): t \geq 0, x \in \Omega\right\}
$$

If $C \subseteq X$ then the dual cone of $C$ is given by

$$
C^{*}=\left\{x^{\prime} \in X^{\prime}:\left\langle x^{\prime}, x\right\rangle \geq 0 \text { for all } x \in C\right\}
$$

([6], I, p. 30).

Psheninchnyi gives a necessary and sufficient condition for a convex programming problem to have a solution in terms of subgradients and the feasible direction cone ([6], II.2.1; [4], 14E). If $f$ is a convex function with $\partial f\left(x_{0}\right)$ a non-void, weak* compact subset, then $f$ attains its minimum at $x_{0} \in \Omega$ if and only if $F\left(\Omega, x_{0}\right) * n \partial f\left(x_{0}\right) \neq 0$.

(Pshenichnyi proves this resurt for $f$ continuous but the continuity is only used to guarantee that $\partial f\left(x_{0}\right)$ is weak * compact and non-empty; actually Pshenichnyi's proof applies to the class of quasi-differentiable

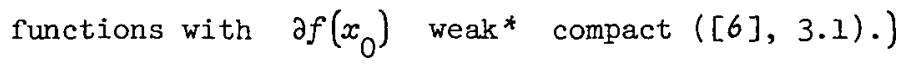

Set $\Omega=\{x: h(x) \leq 0\}$ and let $x_{0} \in \Omega$ be such that $h\left(x_{0}\right)=0$. Concerning the cone $F\left(\Omega, x_{0}\right)$, we have that the inclusion $F\left(\Omega, x_{0}\right) * \supseteq \mathrm{R}_{-} \partial h\left(x_{0}\right)$ holds always, where $\mathrm{R}_{-}$is the set of non-positive real numbers $([6], 2.2 ;[4], 14 E)$. Concerning the equality, we make the following constraint qualification.

DEFINITION 1. The function $h$ is regular at $x_{0}$ if and only if $F\left(\Omega, x_{0}\right) *=\mathrm{R}_{-} \partial h\left(x_{0}\right)$.

A sufficient condition for $h$ to be regular at $x_{0}$ is that $h$ satisfy Slater's condition; that is, there exists $x_{1} \in \Omega$ such that $h\left(x_{1}\right)<0 \quad([6]$, II.2.2; [4], I4E).

From Pshenichnyi's condition given above, we have

THEOREM 2. Let $h$ be regular at $x_{0}$ and let $f$ be such that $\partial f\left(x_{0}\right)$ is weak* compact and non-void. The following conditions are equivalent:

(3) $h(x) \leq 0$ implies $f(x) \geq f\left(x_{0}\right)$; 
(4) there exist $x_{0}^{\prime} \in \partial f\left(x_{0}\right), x_{1}^{\prime} \in \partial h\left(x_{0}\right)$ and $t \leq 0$ such that $x_{0}^{\prime}=t x_{1}^{\prime}$.

Proof. If (3) holds, $x_{0}$ solves the convex programming problem $\min \{f(x): x \in \Omega\}$. By Pshenichnyi's condition and the regularity assumption, (4) follows immediately.

If (4) holds and $x \in \Omega$, then

$$
f(x)-f\left(x_{0}\right) \geq\left\langle x_{0}^{\prime}, x-x_{0}\right\rangle=t\left\langle x_{1}^{\prime}, x-x_{0}\right\rangle \text {. }
$$

But $0 \geq h(x)-h\left(x_{0}\right) \geq\left\langle x_{1}^{\prime}, x-x_{0}\right\rangle$ so $f(x)-f\left(x_{0}\right) \geq 0$, and (3) holds. (This implication does not require regularity.)

This result is given in [4], 14E, under the assumption that $f$ is continuous and Slater's condition is satisfied.

We now indicate haw an infinite dimensional version of the result of Shimizu, Aiyoshi and Katayama can be obtained from Theorem 2. Let $A$ and $B$ be weak* compact subsets of $X^{\prime}$ with $A$ and $B$ such that $\overline{c o} A$ and $\overline{C O} B$ are also weak * compact (this condition is automatically satisfied if $X$ is barrelled $([5], 3.6 .2))$. Set $h=S(\cdot \mid B)=S(\cdot \mid \overline{c o} B)$ and $f=S(\cdot \mid A)=S(\cdot \mid \overline{c o} A)$, where we compute these support functionals in the duality between $X$ and $X^{\prime}$. Note that both $f$ and $h$ are lower semicontinuous and sublinear with $\partial f(0)=\overline{\operatorname{co}} A$. From Theorem 2 we obtain the following generalization of the result of Shimizu, Aiyoshi and Katayama $([7], 2.1)$.

COROLLARY 3. Suppose $0 \neq \overline{\mathrm{CO}} B$. The following conditions are equivalent:

(5) $h(x) \leq 0$ implies $f(x) \geq f(0)=0$;

(6) there exist $x_{0}^{\prime} \in \overline{\operatorname{co}} A, x_{1}^{\prime} \in \overline{\operatorname{co}} B$ and $t \leq 0$ such that $x_{0}^{\prime}=t x_{1}^{\prime}$.

Proof. Since $0 \neq \overline{c o} B$, by the Hahn-Banach Theorem there is an $x_{0} \in X$ such that $h\left(x_{0}\right)=\max \left\{\left\langle x^{\prime}, x_{0}\right\rangle: x^{\prime} \in B\right\}<0$; that is, $h$ satisfies slater's condition. Since $\partial h(0)=\overline{c o} B$ and $\partial f(0)=\overline{c o} A$, Theorem 2 gives the result. 
Shimizu, Aiyoshi and Katayama treat the case when $X=\mathbf{R}^{n}$ is finite dimensional. In this case the weak* topology on $X^{\prime}=\mathbf{R}^{n}$ coincides with the norm topology and if $A, B \subseteq \mathbf{R}^{n}$ are compact, then co $A$ and co $B$ are also compact. Thus, in this case, condition (6) can be stated in stronger form:

(6') there exist $x_{0}^{\prime} \epsilon \operatorname{co} A, x_{1}^{\prime} \epsilon \operatorname{co} B$ and $t \leq 0$ such that $x_{0}^{\prime}=t x_{1}^{\prime}$.

Condition (6') is clearly equivalent to condition (3) of Shimizu, Aiyosh $i$ and Katayama ([7]).

The classical finite dimensional version of the Farkas Lemma corresponds to the case when $B$ is finite and $A$ is a singleton.

The Farkas result of Shimizu, Aiyoshi and Katayama for locally convex spaces has also been obtained by somewhat different methods in [8].

We now also show that a generalized Farkas Lemma of Glover can be obtained from Pshenichnyi's result. Let $f: X \rightarrow \mathbf{R}$ be a lower semicontinuous sublinear function. Let $Y$ be a locally convex space, $S \subseteq Y$ a closed convex cone and $g: X \rightarrow Y$ S-sublinear (that is, $g(t x)=\operatorname{tg}(x)$ for $t \geq 0$ and $-g(t x+(1-t) y)+t g(x)+(1-t) g(y) \in S$ for $0 \leq t \leq 1$, $x, y \in X)$. We assume that $g$ is such that $y^{\prime} g$ is lower semicontinuous on $X$ for each $y^{\prime} \in S^{*}$ (weakly $S^{*}$-lower semicontinuous in Glover's terminology). Glover's result ([3], Theorem 1 ) is given by

THEOREM 4. Let $x^{\prime} \in X^{\prime}$. The following conditions are equivalent:

(7) $-g(x) \in S$ implies that $f(x) \geq\left\langle x^{\prime}, x\right\rangle$;

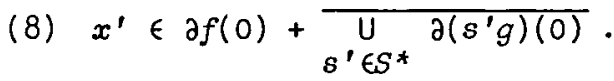

Proof. If $\Omega=g^{-1}(-S)$, then (7) is equivalent to the fact that 0 is a solution of the convex program: $\min \left\{\left(f-x^{\prime}\right)(x): x \in \Omega\right\}$. By Pshenichnyi's condition, since $\partial\left(f-x^{\prime}\right)(0)=\partial f(0)-x^{\prime},(7)$ is equivalent to $\left(\partial f(0)-x^{\prime}\right) \cap F(\Omega, 0) * \neq 0$. But $\Omega$ is a convex cone so $F(\Omega, 0)=\Omega$,

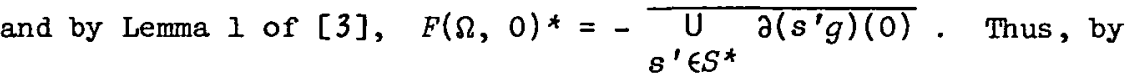

Pshenichnyi's condition, (7) and (8) are equivalent. 


\section{References}

[1] F. Clarke, Optimization and nonsmooth analysis (John Wiley \& Sons, New York and London, 1983).

[2] J. Farkas, "Über die Theorie der einfachen Ungleichen", J. Reine Angew. Math. 124 (1902), 1-24.

[3] B.M. Glover, "A generalized Farkas lemma with applications to quasidifferential programming", Z. Oper. Res. Ser. A 26 (1982), 125-141.

[4] Richard B. Holmes, Geometric functional analysis and its applications (Springer-Verlag, Berlin, Heidelberg, New York, 1975).

[5] John Horvath, Topological vector spaces and distributions, Volume I (Addison-Wesley, Reading, Massachusetts; Palo Alto; London; 1966).

[6] B.N. Pshenichnyi, Necessary conditions for an extremum (Marcel Dekker, New York, 1971).

[7] K. Shimizu, E. Aiyoshi and R. Katayama, "Generalized Farkas' theorem and optimization of infinitely constrained problems", J. Optim. Theory Appl. 40 (1983), 451-462.

[8] Charles Swartz, "A general Farkas lemma", J. Optim. Theory Appl. (to appear).

[9] C. Zalinescu, "A generalization of the Farkas lemma and applications to convex programming", J. Math. Anal. Appl. 66 (1978), 651-678.

[10] C. Zalinescu, "On an abstract control problem", Numer. Funct. Anaz. Optim. 6 (1980), 531-542.

Department of Mathematical Sciences, New Mexico State University,

Las Cruces,

New Mexico 88003,

USA. 